\title{
Smooth particle hydrodynamics in cylindrical coordinates
}

\author{
Leigh Brookshaw*
}

(Received 1 June 2001; revised 9 October 2001)

\section{Abstract}

A derivation of the equations of Smooth Particle Hydrodynamics (SPH) in axisymmetric cylindrical coordinates is presented. The cylindrical SPH formulation is tested by solving two dimensional shock problems.

\section{Contents}

1 Introduction

C115

2 Equations of Motion

2.1 Lagrangian Formulation . . . . . . . . . . C117

\section{Continuity Equation}

${ }^{*}$ Department of Mathematics \& Computing, University of Southern Queensland, Toowoomba, Australia. mailto:leighb@usq.edu.au

${ }^{0}$ See http://anziamj . austms . org. au/V44/CTAC2001/Broo for this article, (C) Austral. Mathematical Soc. 2003. Published 1 April 2003. ISSN 1446-8735 
4 Energy Equation

5 Artificial Viscosity

C122

6 Shock Tube Problem

C123

7 Artificial Thermal Conduction

C129

8 Wall Heating Shocks

C130

9 Conclusion

C137

References

C138

\section{Introduction}

Smoothed Particle Hydrodynamics ( $\mathrm{SPH}$ ) is a Lagrangian method with many attractive features. It is robust, simple to program, and three dimensions are handled as easily as one. SPH is used extensively in astrophysical problems where flows are hydrodynamic, three dimensional and self gravitating [1]. The method can model shocks and has been extended to incorporate material strength with the solution of the stress and strain tensors [2].

The conceptual simplicity of the SPH formulation means that many problems can easily be cast into a three dimensional rectangular Cartesian SPH form. The resolution of a three dimensional SPH calculation is proportional to the cube root of the number of Lagrangian interpolation points used in the problem. This means that computational effort can increase dramatically if a higher resolution is required - in this situation a two dimensional axisymmetric formulation of SPH is required. 
Some axisymmetric SPH algorithms have been formulated [3, 4, $5,6,7]$. None of these formulations seem to have been tested as extensively as the original rectangular Cartesian SPH formulation. The method of Petschek and Libersky [7] is the most self consistent but suffers from a complex formulation and can only work with a Gaussian interpolation kernel, which in general is no longer employed in SPH calculations as it lacks compact support. Apart from the method of Petschek and Libersky none of the other formulations incorporate the hoop stress expected to appear in cylindrical coordinates.

In this paper we turn to a Lagrangian formulation to construct the SPH equations of motion in two dimensional axisymmetric cylindrical coordinates [8]. This method produces equations that naturally conserve energy and contain the expected hoop stress.

\section{Equations of Motion}

The SPH method is a linear interpolation method that can be written in the form

$$
\langle A(\mathbf{s})\rangle=\int_{2 D} A\left(\mathbf{s}^{\prime}\right) W\left(\mathbf{s}-\mathbf{s}^{\prime} ; h\right) d \mathbf{s}^{\prime},
$$

in the cylindrical coordinate system $\mathbf{s}=(r, z)$, where $A(\mathbf{s})$ is the function interpolated, $W$ is the interpolation kernel, and $h$ is the smoothing length of the kernel. As in the standard rectangular Cartesian coordinate system the kernel must mimic a delta function in the limit $h \rightarrow 0$. The most widely used kernel is based on the central B spline $M_{4}$ [9].

If the interpolation points are distributed in the two dimensional 
cylindrical space with a number density $n(\mathbf{s})$ then

$$
\langle A(\mathbf{s})\rangle \approx \sum_{j} \frac{A\left(\mathbf{s}_{j}\right)}{n\left(\mathbf{s}_{j}\right)} W\left(\mathbf{s}-\mathbf{s}_{j} ; h\right) .
$$

If we assign a mass to each interpolation point, then the two dimensional density $\eta$ is

$$
\eta(\mathbf{s})=m n(\mathbf{s})=2 \pi r \rho,
$$

where $\rho$ is the density in three dimensional space. The two dimensional cylindrical density can therefore be calculated directly from the particles by

$$
\eta(\mathbf{s})=\sum_{j} m_{j} W\left(\mathbf{s}-\mathbf{s}_{j} ; h\right)
$$

\subsection{Lagrangian Formulation}

The original energy and momentum conserving formulation of the SPH equations was constructed by starting with a Lagrangian and assuming isentropic flow. This Lagrangian formulation produced a surprising set of SPH equations that have now become the standard. We will follow the same procedure to construct the basic SPH equations in cylindrical coordinates.

The Lagrangian for an isentropic compressible fluid is [10]

$$
L=\int T-E(\rho) d m,
$$

where $T$ is the specific kinetic energy, $E$ is the specific internal energy, and $\rho$ is the density. In the cylindrical coordinates $(r, z)$ the Lagrangian becomes

$$
L=2 \pi \iint\left(\frac{1}{2} \dot{r}^{2}+\frac{1}{2} \dot{z}^{2}-E(\rho)\right) \rho r d r d z,
$$


where a dot over $r$ and $z$ represents the derivative with respect to time $t$ and we have used $d m=2 \pi \rho r d r d z$.

The total kinetic and thermal energies in SPH, are calculated directly from the particles. To use the Lagrangian (6) to derive the SPH equations, the integral must be converted to a summation,

$$
\mathcal{L}=\sum_{j=1}^{N} m_{j}\left(\frac{1}{2} \dot{r}_{j}^{2}+\frac{1}{2} \dot{z}_{j}^{2}-E\left(\rho_{j}\right)\right),
$$

where the summation is over all the particles $i=1, \ldots, N$. The SPH equations of motion are given by Lagrange's equations using the Lagrangian (7) and the fact that the density on particle $i$ at $\mathbf{s}_{i}$ from (4), is

$$
\rho_{i}=\frac{1}{2 \pi r_{i}} \sum_{j=1}^{N} m_{j} W\left(\mathbf{s}_{i}-\mathbf{s}_{j} ; h\right) .
$$

The Lagrangian equation for the $r$-component is

$$
m_{i} \ddot{r}_{i}+\sum_{j} m_{j} \frac{\partial E\left(\rho_{j}\right)}{\partial r_{i}}=0 .
$$

Expanding the summation in $E$ we get

$$
m_{i} \ddot{r}_{i}+m_{i} \frac{\partial E\left(\rho_{i}\right)}{\partial \rho_{i}} \frac{\partial \rho_{i}}{\partial r_{i}}+\sum_{\substack{j=1 \\ j \neq i}}^{N} m_{j} \frac{\partial E\left(\rho_{j}\right)}{\partial \rho_{j}} \frac{\partial \rho_{j}}{\partial r_{i}}=0 .
$$

Using the definition of density, equation (10) becomes

$$
\begin{aligned}
& m_{i} \ddot{r}_{i}+m_{i} \frac{P_{i}}{\rho_{i}^{2}}\left(-\frac{\eta_{i}}{2 \pi r_{i}^{2}}+\frac{1}{2 \pi r_{i}} \sum_{j=1}^{N} m_{j} \frac{\partial W_{i j}}{\partial r_{i}}\right) \\
& \quad+m_{i} \sum_{j=1}^{N} \frac{m_{j}}{2 \pi r_{j}} \frac{P_{j}}{\rho_{j}^{2}} \frac{\partial W_{i j}}{\partial r_{i}}=0,
\end{aligned}
$$


where we have assumed that the fluid is isentropic, $P$ is the pressure, $W_{i j}=W\left(r_{i}-r_{j} ; h\right)$ and the symmetry of the kernel $W_{i j}=W_{j i}$ has been used.

Following the same procedure for the $z$ component, the equations of motion are

$$
\begin{gathered}
\ddot{r}_{i}=2 \pi \frac{P_{i}}{\eta_{i}}-2 \pi \sum_{j=1}^{N} m_{j}\left(\frac{P_{i} r_{i}}{\eta_{i}^{2}}+\frac{P_{j} r_{j}}{\eta_{j}^{2}}\right) \frac{\partial W_{i j}}{\partial r_{i}}, \\
\ddot{z}_{i}=-2 \pi \sum_{j=1}^{N} m_{j}\left(\frac{P_{i} r_{i}}{\eta_{i}^{2}}+\frac{P_{j} r_{j}}{\eta_{j}^{2}}\right) \frac{\partial W_{i j}}{\partial z_{i}} .
\end{gathered}
$$

The first term of equation (12) is the term that provides the 'hoop' stress. This stress appears in the equations because an SPH interpolation point can be viewed as a "smeared out" particle or mass hoop with an internal pressure. This means that the pressure force on the outside surface of the hoop, or the surface with the greater radius, is larger than the inner surface, or the surface with the smaller radius. This produces a net outward force on the SPH mass 'hoop'.

These equations are similar in structure to the Cartesian SPH equations. Like the rectangular Cartesian SPH equations they can also be constructed without recourse to a Lagrangian (in hindsight). Starting with the equation of motion for the coordinate $r$,

$$
\ddot{r}=-\frac{1}{\rho} \frac{\partial P}{\partial r},
$$

we first note

$$
\frac{\partial P}{\partial r}=\frac{1}{r}\left(\frac{\partial(r P)}{\partial r}-P\right),
$$

and

$$
\frac{1}{\eta} \frac{\partial(r P)}{\partial r}=\frac{\partial}{\partial r}\left(\frac{r P}{\eta}\right)+\frac{r P}{\eta^{2}} \frac{\partial \eta}{\partial r} .
$$


Combining both equations, we get

$$
\ddot{r}=-\frac{1}{\rho} \frac{\partial P}{\partial r}=2 \pi \frac{P}{\eta}-2 \pi\left(\frac{\partial}{\partial r}\left(\frac{r P}{\eta}\right)+\frac{r P}{\eta^{2}} \frac{\partial \eta}{\partial r}\right) .
$$

This equation, when converted into an SPH interpolation form, will give equation (12). Equation (13) follows similarly.

\section{Continuity Equation}

For exact mass conservation the continuity equation can be replaced with the density interpolant equation (4). For some problems however, the continuity equation needs to be solved explicitly.

The continuity equation, in the cylindrical coordinates $(r, z)$ is

$$
\frac{\partial \rho}{\partial t}+\frac{\rho}{r} \frac{\partial}{\partial r}(r \dot{r})+\rho \frac{\partial \dot{z}}{\partial z}=0 .
$$

It can be rewritten in terms of the two dimensional density $\eta$, such that

$$
\frac{\partial \eta}{\partial t}+\mathbf{D} \cdot(\eta \dot{\mathbf{s}})-\dot{\mathbf{s}} \cdot \mathbf{D} \eta=0,
$$

where $\dot{\mathbf{s}}=(\dot{r}, \dot{z})$ and the differential operator $\mathbf{D}$ is defined as

$$
\mathbf{D}=\hat{\mathbf{r}} \frac{\partial}{\partial r}+\hat{\mathbf{z}} \frac{\partial}{\partial z}
$$

This equation, converted into an SPH form, becomes

$$
\frac{\partial \eta_{i}}{\partial t}=\sum_{j=1}^{N} m_{j}\left(\dot{\mathbf{s}}_{i}-\dot{\mathbf{s}}_{j}\right) \cdot \mathbf{D}_{i} W_{i j} .
$$




\section{Energy Equation}

In the absence of sources and sinks the rate of change of the specific thermal energy $E$ is

$$
\begin{aligned}
\frac{\mathrm{d} E}{\mathrm{~d} t} & =-\frac{P}{\rho} \nabla \cdot \dot{\mathbf{s}} \\
& =-\frac{P}{r \rho} \dot{r}-\frac{P}{\rho} \mathbf{D} \cdot \dot{\mathbf{s}} .
\end{aligned}
$$

There are many ways this equation can be written in a form suitable for SPH. For example, starting with the identity

$$
\frac{P r}{\eta} \mathbf{D} \cdot \dot{\mathbf{s}}=\mathbf{D} \cdot\left(\frac{P r \dot{\mathbf{s}}}{\eta}\right)-\dot{\mathbf{s}} \cdot \mathbf{D}\left(\frac{P r}{\eta}\right),
$$

we find the following SPH equation for the energy of particle $i$

$$
\frac{\mathrm{d} E_{i}}{\mathrm{~d} t}=-2 \pi \frac{P_{i}}{\eta_{i}} \dot{r}_{i}+2 \pi \sum_{j=1}^{N} m_{j} \frac{P_{j} r_{j}}{\eta_{j}^{2}}\left(\dot{\mathbf{s}}_{i}-\dot{\mathbf{s}}_{j}\right) \cdot \mathbf{D}_{i} W_{i j} .
$$

Using the identity

$$
\eta \mathbf{D} \cdot \mathbf{v}=\mathbf{D} \cdot(\dot{\mathbf{s}} \eta)-\dot{\mathbf{s}} \cdot \mathbf{D} \eta,
$$

we get the alternate SPH energy equation

$$
\frac{\mathrm{d} E_{i}}{\mathrm{~d} t}=-2 \pi \frac{P_{i}}{\eta_{i}} \dot{r}_{i}+2 \pi \frac{P_{i} r_{i}}{\eta_{i}^{2}} \sum_{j=1}^{N} m_{j}\left(\dot{\mathbf{s}}_{i}-\dot{\mathbf{s}}_{j}\right) \cdot \mathbf{D}_{i} W_{i j} .
$$

Or combining both equations

$$
\frac{\mathrm{d} E_{i}}{\mathrm{~d} t}=-2 \pi \frac{P_{i}}{\eta_{i}} \dot{r}_{i}+\pi \sum_{j=1}^{N} m_{j}\left(\frac{P_{i} r_{i}}{\eta_{i}^{2}}+\frac{P_{j} r_{j}}{\eta_{j}^{2}}\right)\left(\dot{\mathbf{s}}_{i}-\dot{\mathbf{s}}_{j}\right) \cdot \mathbf{D}_{i} W_{i j}
$$


The first term in the SPH energy equation is the energy change associated with the 'hoop' stress.

In the tests that follow equation (28) was used throughout and energy was found to be conserved to five significant figures.

\section{Artificial Viscosity}

If shock phenomena is to be modelled using the SPH equations then an artificial viscosity must be added to the equations to dampen post shock oscillations in the solution and to prevent particle interpenetration in high Mach number collisions.

We follow the rectangular Cartesian SPH formulation of [11] and [12] by writing the momentum equations as

$$
\frac{\mathrm{d} \dot{\mathbf{s}}_{i}}{\mathrm{~d} t}=2 \pi \frac{P_{i}}{\eta_{i}} \mathbf{D}_{i} r_{i}-2 \pi \sum_{j=1}^{N} m_{j}\left(\frac{P_{i} r_{i}}{\eta_{i}^{2}}+\frac{P_{j} r_{j}}{\eta_{j}^{2}}\right)\left(1-\alpha \mu_{i j}+\beta \mu_{i j}^{2}\right) \mathbf{D}_{i} W_{i j},
$$

where $\alpha$ and $\beta$ are non-dimensional constants, and

$$
\mu_{i j}= \begin{cases}\frac{h \dot{\mathbf{s}}_{i j} \cdot \mathbf{s}_{i j}}{c\left(s_{i j}^{2}+\zeta^{2}\right)}, & \dot{\mathbf{s}}_{i j} \cdot \mathbf{s}_{i j}<0 \\ 0, & \dot{\mathbf{s}}_{i j} \cdot \mathbf{s}_{i j}>0\end{cases}
$$

where $c$ is the maximum sound speed, and $\mathbf{s}_{i j}=\mathbf{s}_{i}-\mathbf{s}_{j}$.

The viscosity is zero for particles moving away from each other and positive for particles approaching each other.

Following the procedure in [13], it is easy to show in the limit 
as $N \rightarrow \infty$ and $h \rightarrow 0$, equation (29) becomes in one dimension

$$
\frac{\mathrm{d} \dot{r}}{\mathrm{~d} t}=-\frac{1}{\rho} \frac{\partial P}{\partial r}+\frac{\alpha h}{c r \rho} \frac{\partial}{\partial r}\left(r P \frac{\partial \dot{r}}{\partial r}\right)-\frac{\beta}{r \rho}\left(\frac{h}{c}\right)^{2} \frac{\partial}{\partial r}\left(r P\left(\frac{\partial \dot{r}}{\partial r}\right)^{2}\right),
$$

which shows that the effect of the viscosity term is to add a bulk viscosity and a von Neumann-Richtmyer viscosity to the equation of motion. The bulk viscosity dampens post shock oscillations, and the von Neumann-Richtmyer viscosity is required for high Mach number collisions to stop inter-penetration of particles.

The introduction of a viscous term in the equations of motion means that for energy conservation the term must also be introduced into the energy equation. Therefore the energy equation becomes

$$
\begin{aligned}
& \frac{\mathrm{d} E_{i}}{\mathrm{~d} t}=-2 \pi \frac{P}{\eta} \dot{r}_{i} \\
& +\pi \sum_{j=1}^{N} m_{j}\left(\frac{P_{i} r_{i}}{\eta_{i}^{2}}+\frac{P_{j} r_{j}}{\eta_{j}^{2}}\right)\left(1-\alpha \mu_{i j}+\beta \mu_{i j}^{2}\right)\left(\dot{\mathbf{s}}_{i}-\dot{\mathbf{s}}_{j}\right) \cdot \mathbf{D}_{i} W_{i j} .
\end{aligned}
$$

\section{Shock Tube Problem}

Consider a cylindrical version of the linear shock tube problem for a perfect gas. The initial conditions are:

$$
\begin{array}{lll}
r<5, & \rho=1, & E=1, \\
r>5, & \rho=0.25, & E=1,
\end{array}
$$

with the constitutive equation

$$
P=(\gamma-1) \rho E,
$$


and with the ratio of specific heats $\gamma=1.4$.

For the SPH simulation we distribute the particles with separation $\delta r=0.01$ in the low density region and with $\delta r=0.0025$ in the high density region. The smoothing length is constant throughout the domain at $h=0.02$. To model a constant density fluid with equally separated particles the mass of each particle must be proportional to $r$. No special boundary conditions are applied at the end points, as the rarefaction wave produced does not have time to propagate inward over the time of the simulation. The smoothing length is held constant throughout the calculation.

The time step $\delta t$ is limited by the Courant condition, and by the viscous diffusion introduced by the artificial viscosity. The criterion used in [12] seems to lead to a stable algorithm at all radii.

The SPH calculation is compared to a Lagrangian finite difference simulation $[14,15]$. The finite difference code uses a grid with $\delta r=0.002$. The finite difference code, like the SPH code, has been stabilised to post shock oscillations by the inclusion of a bulk and von Neumann-Richtmyer artificial viscosity. (Following Noh [15] the bulk viscosity constant is $C_{1}=2$, and the von Neumann-Richtmyer constant $C_{0}^{2}=2$.)

The finite difference and the SPH schemes have error terms of second order, which implies that the finite difference scheme will have a truncation error two orders of magnitude smaller than the SPH method.

Figures 1, 2, 3, and 4 show the density, specific thermal energy, radial velocity and the pressure profiles respectively. The SPH profiles compare favourably with the more accurate finite difference results. The shock front and contact discontinuity are broadened over $2 h-3 h$ as is expected with the inclusion of an artificial viscosity. The oscillations in the velocity profile are negligible. The 


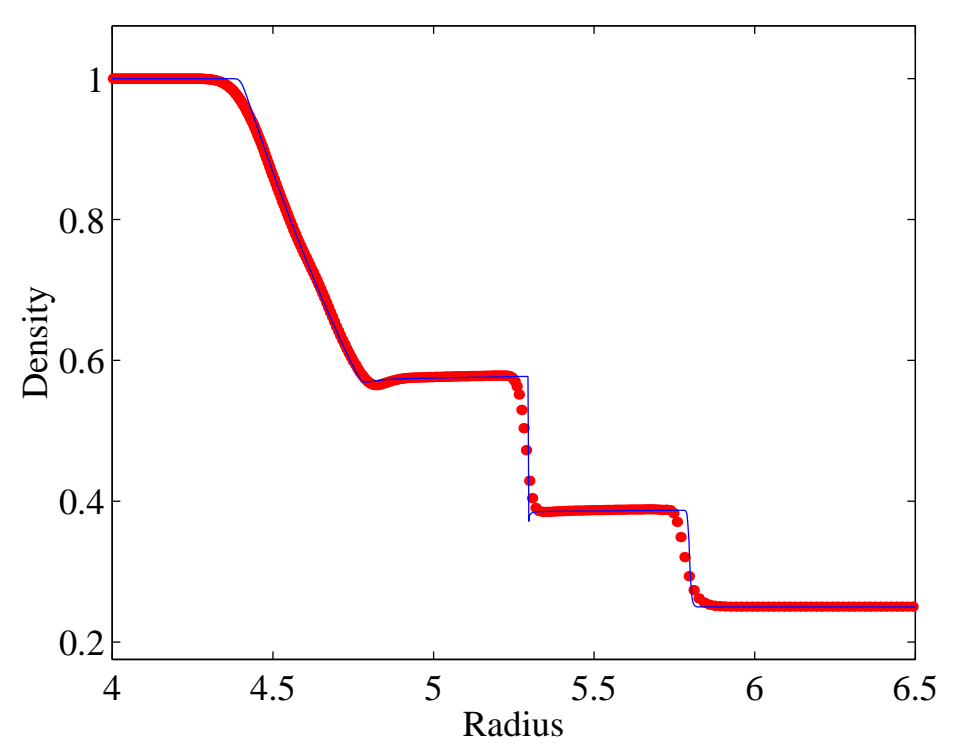

Figure 1: Density profile of the shock expansion problem described in Section 6. The SPH result $(\cdots)$ has $h=0.02, \alpha=4.0$ and $\beta=$ 2.0. The Lagrangian finite difference result $(-)$ has $\delta r=0.002$. 


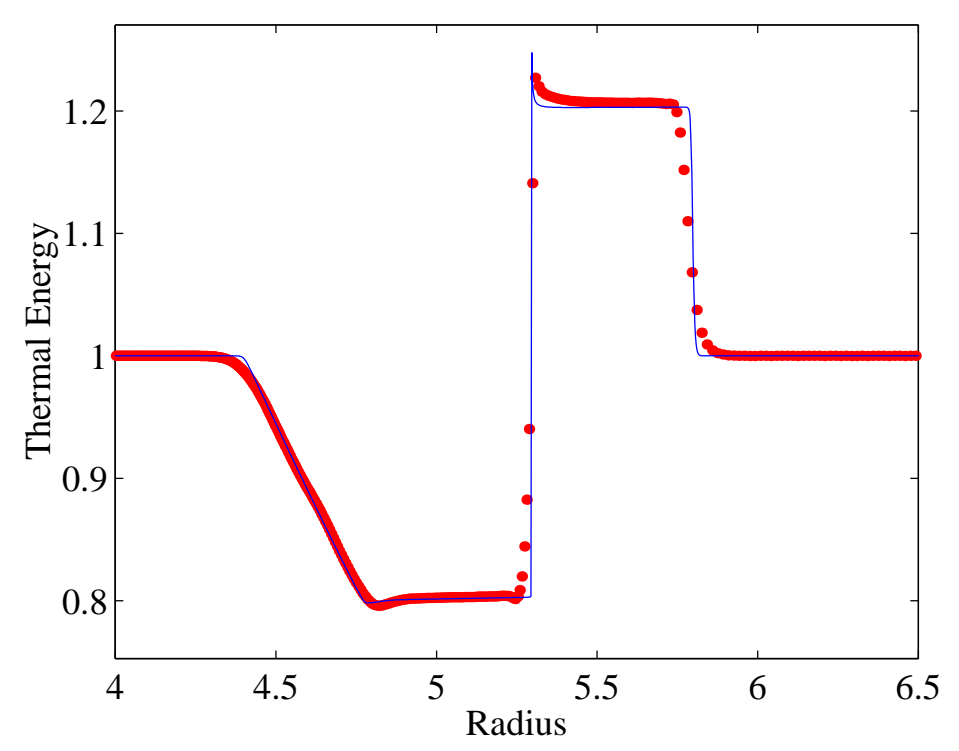

FiguRE 2: Thermal energy profile of the shock expansion problem described in Section 6. The SPH result $(\cdots)$ has $h=0.02, \alpha=4.0$ and $\beta=2.0$. The Lagrangian finite difference result $(-)$ has $\delta r=$ 0.002 . 


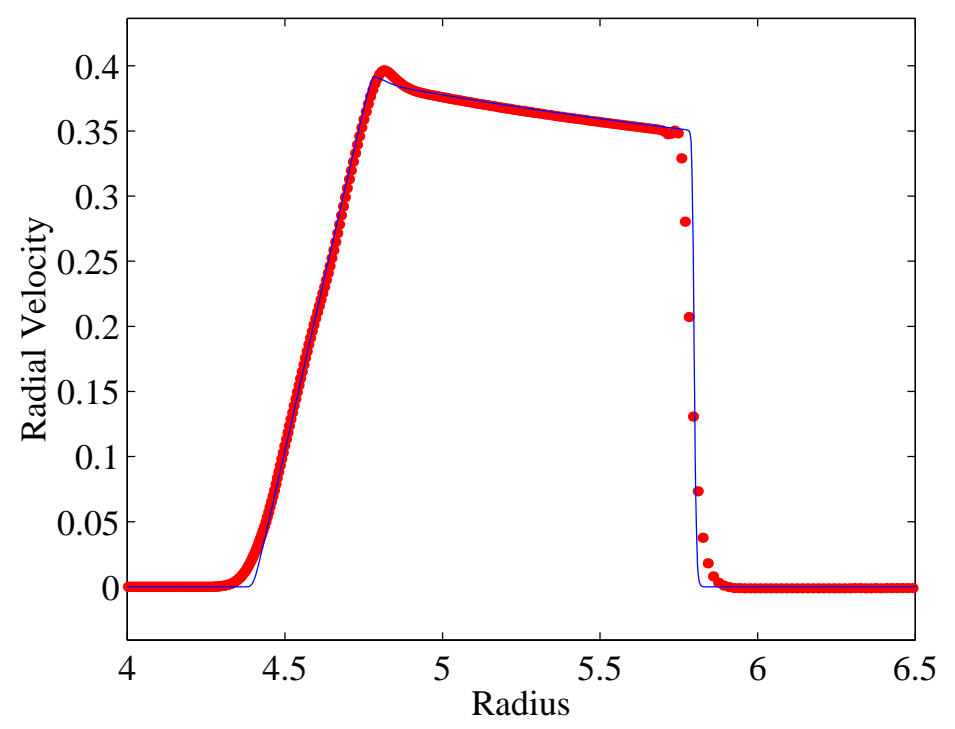

Figure 3: Radial velocity profile of the shock expansion problem described in Section 6. The SPH result $(\cdots)$ has $h=0.02, \alpha=4.0$ and $\beta=2.0$. The Lagrangian finite difference result $(-)$ has $\delta r=$ 0.002 . 


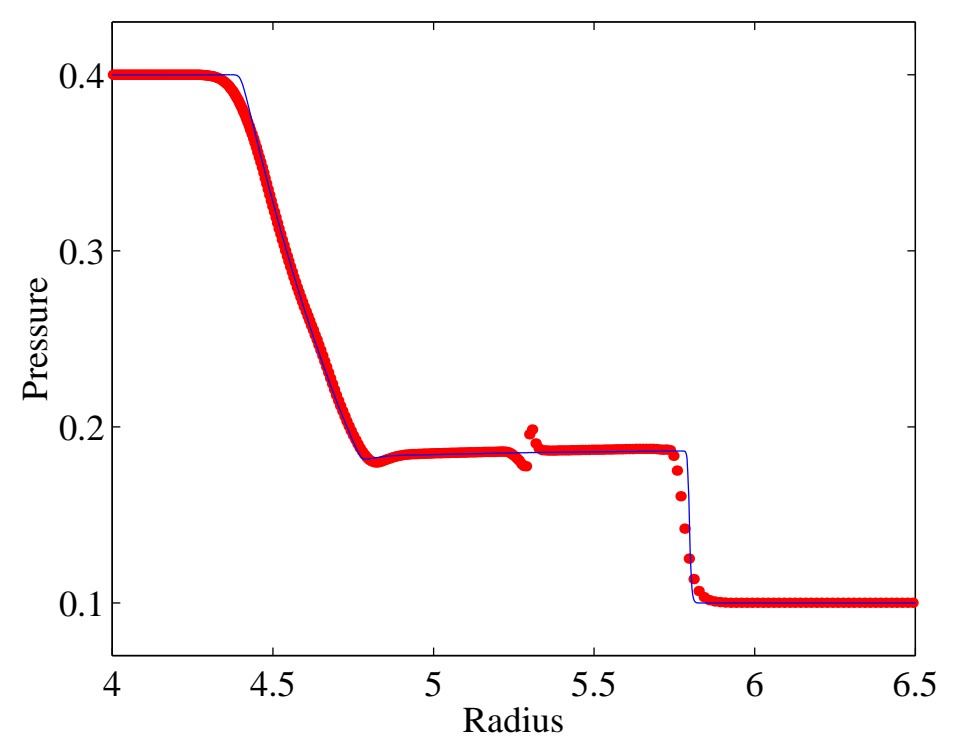

Figure 4: Pressure profile of the shock expansion problem described in Section 6. The SPH result $(\cdots)$ has $h=0.02, \alpha=4.0$ and $\beta=2.0$. The Lagrangian finite difference result $(-)$ has $\delta r=0.002$. 
thermal energy exhibits the largest error at the contact discontinuity. The error also appears in the finite difference calculation. This error appears to be an example of Noh's 'wall heating' [15], (see $\S 7)$. The error in the thermal energy appears in the pressure as the break at the contact discontinuity.

Tests at different radii show that the broadening of the shock and contact discontinuities, and the viscosity constants appear to be independent of the radius.

\section{Artificial Thermal Conduction}

The introduction of an artificial viscosity, in Lagrangian methods, can introduce errors in the form of excessive heating when streams of gas collide, or when a stream of gas is brought to rest against a wall (hence the term 'wall heating'). Noh [14, 15] has shown that if an artificial thermal diffusion is introduced into the equations the excessive heating can be reduced significantly. The heat conduction term also helps to smooth errors in the velocity field which can lead to a reduction in the artificial viscosity and a sharpening of the shock. Following Monaghan [16], and Noh [14, 15] the thermal energy equation needs to be modified by the inclusion of a term of the form

$$
\frac{1}{\rho} \boldsymbol{\nabla} \cdot(H \nabla E) \text {. }
$$

This term is estimated by the following SPH expression:

$$
\sum_{j=1}^{N} \frac{H_{i j}}{\bar{\eta}_{i j}} \frac{\left(E_{i}-E_{j}\right)}{\left(\mathbf{s}_{i j}^{2}+\zeta^{2}\right)} \mathbf{s} \cdot \mathbf{D}_{i} W_{i j},
$$

where $\bar{\eta}_{i j}=\left(\eta_{i}+\eta_{j}\right) / 2$ and

$$
H_{i j}=g_{1} h \bar{c}_{i j}+g_{2} h^{2}\left|\mu_{i j}\right|,
$$


where $\bar{c}_{i j}=\left(c_{i}+c_{j}\right) / 2$, and $g_{1}$ and $g_{2}$ are constants.

In the limit as $N \rightarrow \infty$ and $h \rightarrow 0$, equation (35) becomes in one dimension

$$
\frac{1}{r \rho} \frac{\partial}{\partial r}\left(H r \frac{\partial E}{\partial r}\right),
$$

where

$$
H=g_{1} h \rho c+g_{2} \rho h^{2} \frac{\partial \dot{r}}{\partial r} .
$$

Equation (35) when added to the SPH energy equation behaves as a heat conduction term, that adds nothing to the overall energy of the system. To control 'wall heating' only, equation (35) is added to those particles with $\dot{\mathbf{s}}_{i j} \cdot \mathbf{s}_{i j}<0$, that is where the artificial viscosity is non-zero. The conduction coefficient $H$ is made up of two terms, the first is a linear term that is a function of the local sound speed, the second term is a quadratic term that is a function of the local velocity gradient. The second term will dominate in the vicinity of a shock.

\section{Wall Heating Shocks}

To investigate the effects of 'wall heating' we model two streams of gas impacting with a relative velocity greater than their sound speed. The initial conditions are:

$$
\begin{array}{lll}
r<3, & \rho=1, \quad E=1, \quad \dot{r}=1, \\
r>3, & \rho=1, \quad E=1, \quad \dot{r}=-1,
\end{array}
$$

where, with the ratio of specific heats $\gamma=1.4$ the sound speed of the undisturbed gas is $c=0.748$. 


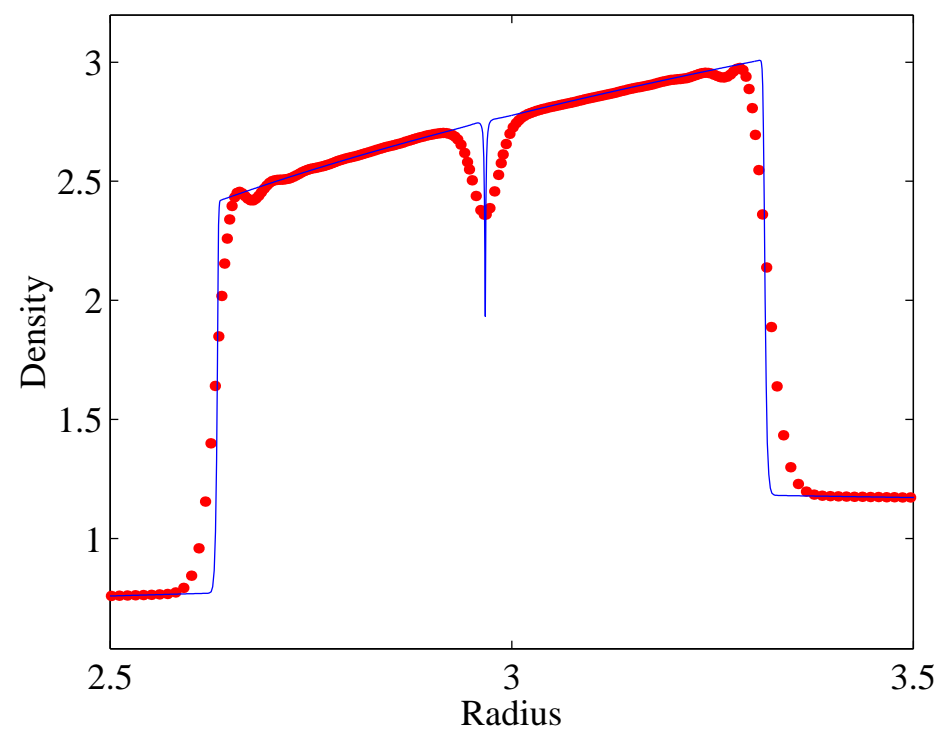

FiguRE 5: Density profile of the shock collision problem described in Section 7 without heat conduction $\left(g_{1}=g_{2}=0\right)$. The SPH result $(\cdots)$ has $h=0.02, \alpha=5.0$ and $\beta=5.0$. The Lagrangian finite difference result $(-)$ has $\delta r=0.002$.

The particles were distributed with a separation of $\delta r=0.01$ and with a smoothing length $h=0.02$. The smoothing length is held constant throughout the calculation.

Figures 5, 6, and 7 show the density, specific thermal energy, and radial velocity profiles respectively. This run shows the results when both the SPH and the finite difference calculation do not have a heat conduction term incorporated into the energy equation. The spike in the energy profile, at the point of impact is quite clear. The effect of the spike in the energy is to cause a dip in the density. Increasing the artificial viscosity will not remove the spike, but does 


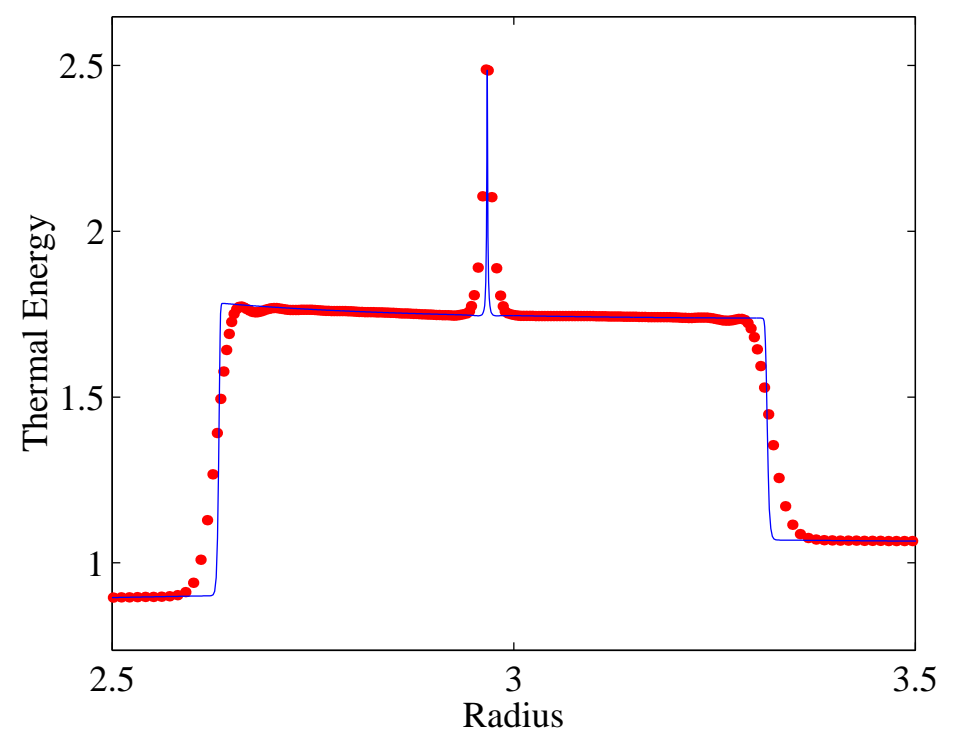

Figure 6: Thermal energy profile of the shock expansion problem described in Section 7 without heat conduction $\left(g_{1}=g_{2}=0\right)$. The SPH result $(\cdots)$ has $h=0.02, \alpha=5.0$ and $\beta=5.0$. The Lagrangian finite difference result $(-)$, has $\delta r=0.002$. 


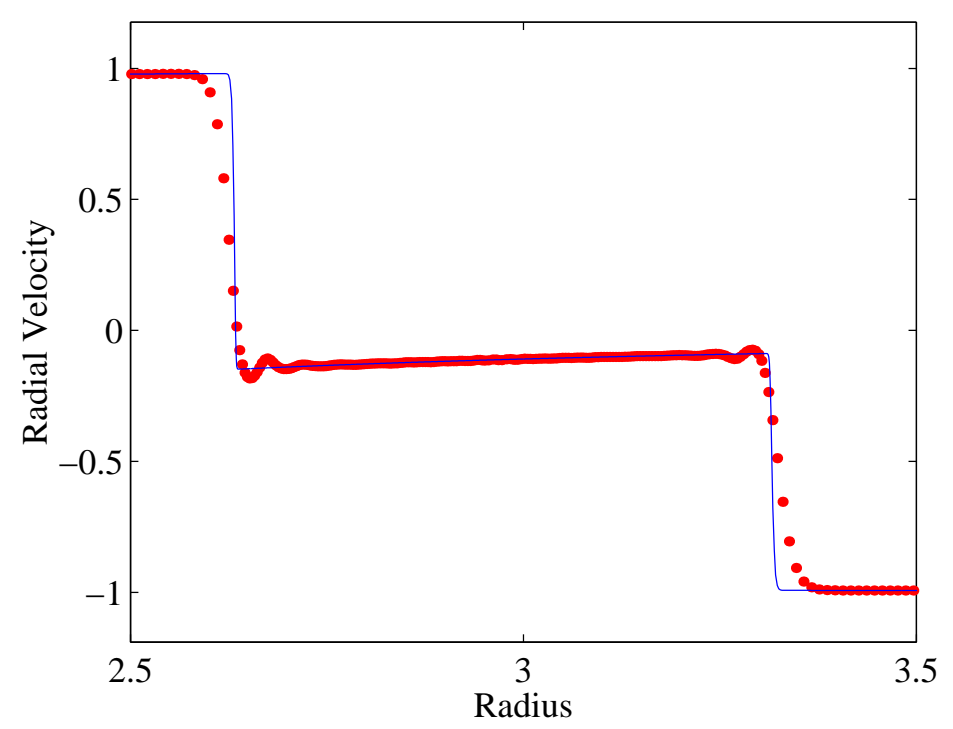

FiguRE 7: Radial velocity profile of the shock expansion problem described in Section 7 without heat conduction $\left(g_{1}=g_{2}=0\right)$. The SPH result $(\cdots)$ has $h=0.02, \alpha=5.0$ and $\beta=5.0$. The Lagrangian finite difference result $(-)$ has $\delta r=0.002$. 


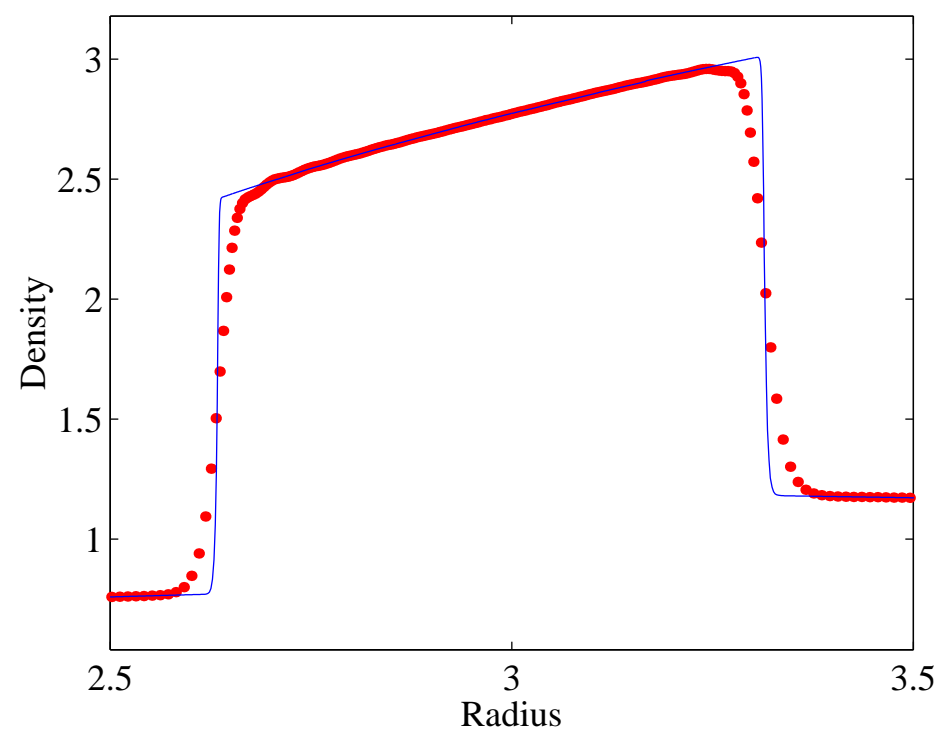

FiguRE 8: Density profile of the shock collision problem described in Section 7 with heat conduction. The SPH result $(\cdots)$ has $h=$ $0.02, \alpha=3.0, \beta=3.0, g_{1}=1.0$, and $g_{2}=2.0$. The Lagrangian finite difference result $(-)$ has $\delta r=0.002$.

broaden the shock. Decreasing the artificial viscosity will reduce the spike, but oscillations in the profiles increase. The spike is a direct result of the inclusion of an artificial viscosity into the equations.

Figures 8, 9, and 10 show the density, specific thermal energy, and radial velocity profiles respectively, when artificial heat conduction is added to the thermal energy equation of both methods. The plots show the profiles at the same time as Figures 5, 6, and 7. With the inclusion of the artificial conduction $\left(g_{1}=1, g_{2}=2\right)$, the artificial viscosity was reduced slightly $(\alpha=3, \beta=3)$. The effect of the artificial heat conduction is to remove the spike in the 


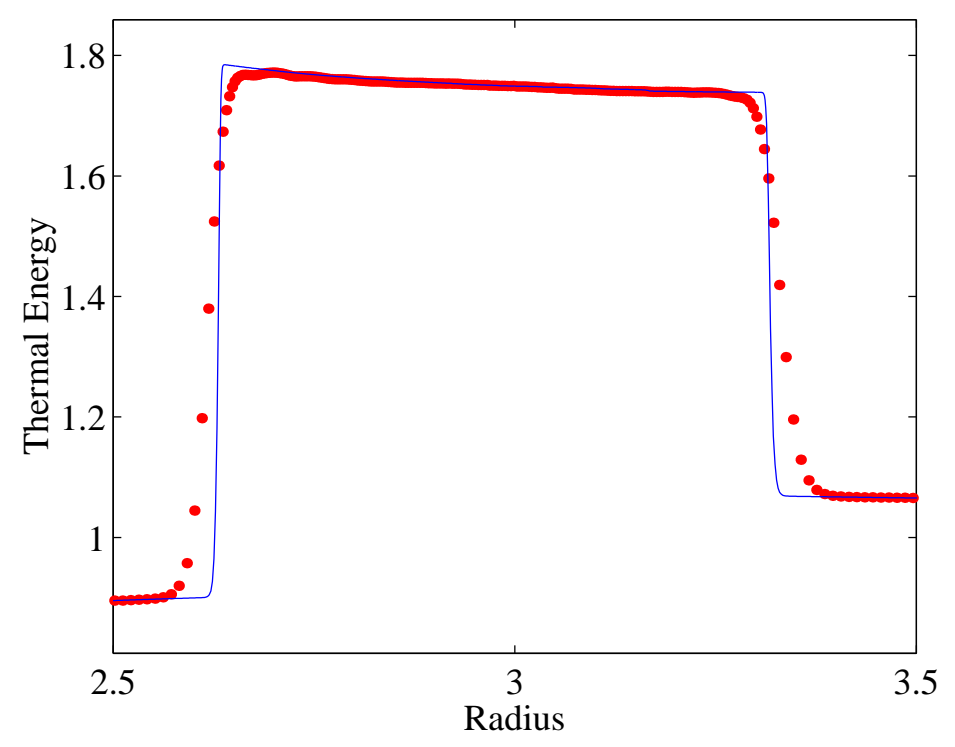

Figure 9: Thermal energy profile of the shock expansion problem described in Section 7 with heat conduction. The SPH result $(\cdots)$ has $h=0.02, \alpha=3.0, \beta=3.0, g_{1}=1.0$, and $g_{2}=2.0$. The Lagrangian finite difference result $(-)$ has $\delta r=0.002$. 


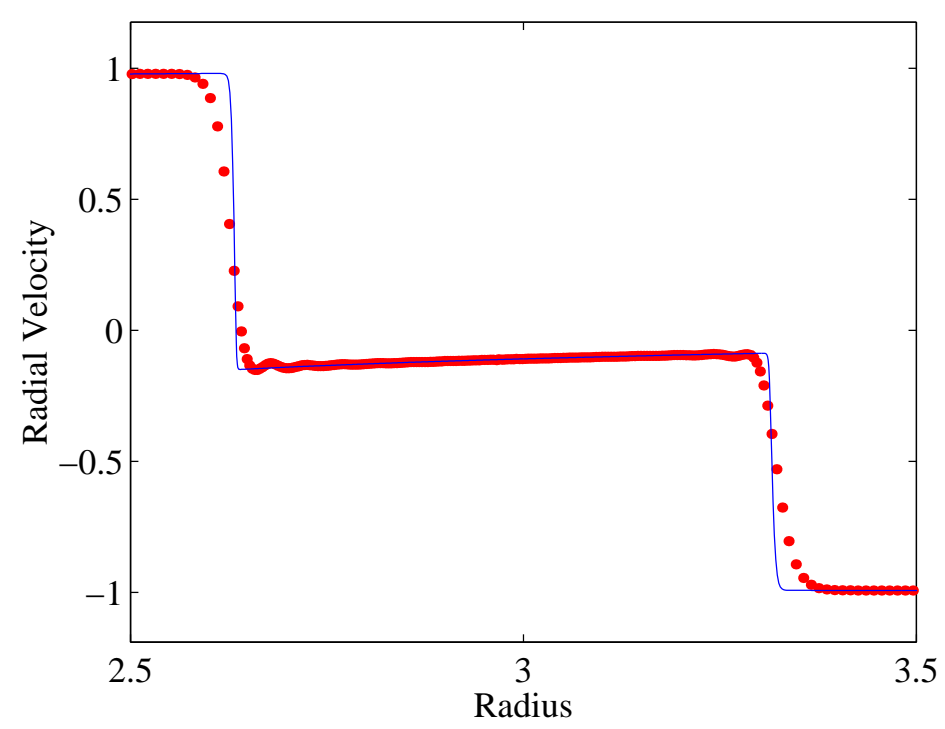

FiguRE 10: Radial velocity profile of the shock expansion problem described in Section 7 without heat conduction. The SPH result $(\cdots)$ has $h=0.02, \alpha=3.0$ and $\beta=3.0, g_{1}=1.0$, and $g_{2}=2.0$. The Lagrangian finite difference result $(-)$ has $\delta r=0.002$. 
energy completely. The broadening of the shock has not changed, but the shoulder of the shock has become rounder, with the small oscillations in density and velocity removed.

The effect of the inclusion of an artificial heat conduction in the finite difference equation is to remove the thermal energy spike and to sharpen the shock. In the SPH formulation, though the artificial viscosity can be reduced there is little evidence that the shock is sharpened by the inclusion of the artificial heat conduction. If anything the rounding of the shoulders of the shock can give the effect of broadening the shock.

A small amount of artificial heat conduction added to the energy equation for the shock tube result $\left(g_{1}=0.2, g_{2}=0\right)$ can greatly reduce the error at the contact discontinuity. But care has to be taken as the contact discontinuity is sensitive to the heat conduction and can be greatly reduced. As expected the contact discontinuity is sensitive only to the linear heat conduction term not the quadratic term.

\section{Conclusion}

This study shows that the cylindrical SPH formulation presented, performs as well as the rectangular SPH formulation for the modelling of shocks. The artificial viscosity and artificial heat conduction perform as well as their rectangular Cartesian counterparts.

The advantages of the cylindrical SPH formulation presented here is that it is very similar in structure, derivation and errors to its rectangular counterpart.

What has not been presented here, and is currently under inves- 
tigation is a self consistent SPH formulation within the vicinity $\sim 2 h$, of the line of symmetry $r=0$.

\section{References}

[1] Monaghan J.J., Smoothed Particle Hydrodynamics, Annu. Rev. Astron. Astrophys., 30:543-74, 1992. C115

[2] Libersky L.D., Petschek A.G., Smooth Particle Hydrodynamics with strength of materials. In Lecture Notes in Physics 395: Advances in the Free-Lagrange Method, (Ed. Trease, Fritts, Crowley), Springer-Verlag, pp248-257, 1991. C115

[3] Coleman, C.S., and Bicknell, G.V., Jets with entrained clouds - I. Hydrodynamic simulations and magnetic field structure, Mon. Not. R. astr. Soc., 214:337-355, 1985. C116

[4] Stellingwerf, R.F., Smooth Particle Hydrodynamics. In Lecture Notes in Physics 395: Advances in the Free-Lagrange Method, (Ed. Trease, Fritts, Crowley), Springer-Verlag, pp239-248, 1991. C116

[5] Herant, M., and Benz, W., Postexplosion Hydrodynamics of SN 1987A, Astrophys. J., 387:294-308, 1992. C116

[6] Chakrabarti, S.K., and Molteni, D., Smoothed Particle Hydrodynamics Confronts Theory: Formation of Standing Shocks in Accretion Disks and Wind Around Black Holes, Astrophys. J., 417:671-676, 1993. C116

[7] Petscheck, A.G., and Libersky L.D., Cylindrical Smoothed Particle Hydrodynamics, J. Comput. Phys., 109:76-83, 1993. C116 
[8] Gingold, R.A., and Monaghan, J.J., Kernel Estimates as a Basis for General Particle Methods in Hydrodynamics, J. Comput. Phys., 46:429-453, 1982. C116

[9] Monaghan, J.J., Particle Methods for Hydrodynamics, Computer Physics Reports, 3:71-124, 1985. C116

[10] Eckert, C., Variation Principles of Hydrodynamics, Physics of Fluids, 3(3):421-427, 1960. C117

[11] Monaghan, J.J., and Pongracic, H., Artificial Viscosity for Particle Methods, IMACS J. Num. Math., 1:187-194, 1985. C122

[12] Lattanzio J.C., Monaghan, J.J., Pongracic, H., and Schwartz, M.P. Controlling Penetration, SIAM J. Sci. Stat. Comput.., 7(2):591-598, 1986. C122, C124

[13] Monaghan, J.J., and Gingold, R.A., Shock Simulation by the Particle Method SPH, J. Comput. Phys., 52:374-389, 1983. C122

[14] Noh, W.F., Artificial Viscosity (Q) and Artificial Heat Flux (H) Errors for Spherically Divergent Shocks, Lawrence Livermore National Laboratory technical report UCRL-89623, 1983. C124, C129

[15] Noh, W.F., Errors for Calculations of Strong Shocks Using an Artificial Viscosity and an Artificial Heat Flux, J. Comput. Phys., 72:78-120, 1987. C124, C129

[16] Monaghan, J.J., SPH meets the Shocks of Noh, Monash University preprint, 1988. C129 